\title{
Frecuencia de los genotipos babA2, oipA y cagE de Helicobacter pylori en pacientes colombianos con enfermedades gastroduodenales
}

\author{
Andrés Javier Quiroga, Diana Marcela Cittelly, María Mercedes Bravo \\ Laboratorio de Inmunología, Instituto Nacional de Cancerología, Bogotá, D. C., Colombia. \\ Introducción. La infección con Helicobacter pylori está asociada con el desarrollo de diferentes \\ enfermedades gastroduodenales. Varios genes de virulencia de $H$. pylori se han relacionado \\ con mayor riesgo de enfermedad gástrica. \\ Objetivos. El propósito de este trabajo fue determinar las posibles asociaciones entre la \\ presencia de los genes vacA, cagA, cagE, babA2 y oipA en aislamientos de $H$. pylori de \\ pacientes colombianos y las diferentes consecuencias clínicas de la infección. \\ Materiales y métodos. Mediante PCR se evaluaron los genotipos cagA, vacA, cagE, oipA y \\ babA2 en 166 aislamientos de $H$. pylori provenientes de 50 pacientes con úlcera péptica, 39 \\ con gastritis crónica no atrófica, 26 con gastritis crónica atrófica, 26 con metaplasia intestinal y \\ 25 con adenocarcinoma gástrico. \\ Resultados. La frecuencia de los genotipos cagA, cagE, babA2 y oipA fue de $73 \%, 75 \%, 48 \%$ \\ y $74 \%$, respectivamente. El 64\% (100/157) de los aislamientos presentó el genotipo citotóxico \\ vacAs1m1/cagA positivo/cagE positivo. Se observó una mayor frecuencia de cepas citotóxicas \\ en pacientes con cáncer (84\%), metaplasia (91\%) y úlcera (81\%) en comparación con pacientes \\ con gastritis no atrófica (50\%) $(p=0,002,0,008$ y 0,007 , respectivamente). La frecuencia de \\ oipA y babA2 fue mayor en cepas citotóxicas que en cepas no citotóxicas (oipA: $81 \%$ vs. $52 \%$, \\ $p=0,003$; babA2: $58 \%$ vs. $12 \%, p=0,000$ ). No se observaron diferencias significativas en la \\ frecuencia de los genes oipA o babA2 solos o en asociación con vacA y cagA/cagE y las \\ diferentes enfermedades gastroduodenales. \\ Conclusiones. No se encontraron evidencias que sugieran que los genes babA2 u oipA \\ puedan servir como marcadores de ulcerogénesis o carcinogénesis en esta población, solos \\ o en asociación con cagA, cagE o vacA.
}

Palabras clave: Helicobacter pylori, genotipo, enfermedades del sistema digestivo, Colombia.

BabA2, oipA and cagE Helicobacter pylori genotypes in Colombian patients with gastroduodenal diseases

Introduction. Helicobacter pylori infection is associated with the development of several gastroduodenal diseases. Bacterial virulence genes have been found associated with an increased risk for gastric disease.

Objectives. Herein, associations were made between the presence of $\operatorname{vac} A$, $\operatorname{cag} A$, $\operatorname{cag} E$, babA2 and oipA genes in $H$. pylori isolates and the range of clinical consequences of the infection.

Methods. PCR was used to amplify vacA, cagA, cagE, babA2 and oipA genes in 166 isolates50 patients with peptic ulcer, 39 with non-atrophic gastritis, 26 with atrophic gastritis, 26 with intestinal metaplasia and 25 with gastric adenocarcinoma.

Results. cagA, cagE, babA2 and oipA genes were found in $73 \%, 75 \%, 48 \%$ and $74 \%$ of isolates, respectively. The cytotoxic vacA $\mathrm{s} 1 \mathrm{~m} 1 /$ cagA positive/cage positive genotype was present in $64 \%(100 / 157)$ of isolates. A higher frequency of cytotoxic strains was observed in cancer patients (84\%), intestinal metaplasia (91\%) and peptic ulcer (81\%) in comparison with gastritis patients $(50 \%)(p=0.002,0.008,0.007$, respectively). The oipA and babA2 frequency was higher in cytotoxic isolates than in non-cytotoxic isolates (oipA: $81 \%$ vs. $52 \%, \mathrm{P}=0,003 ;$ babA2: $58 \%$ 
vs. $12 \%$ ( $p<0.001)$. No significant association was found among clinical outcomes and oipA or babA2 genotypes, analyzed alone or in combination with vacA and cagA.

Conclusion. Therefore, babA2 or oipA genes are not marker indicators of ulcer or cancer.

Keywords: Helicobacter pylori, genotype, digestive system diseases, Colombia.

Helicobacter pylori ha sido considerado como carcinógeno humano por la International Agency for research on cancer (1). Se estima que más de la mitad de la población mundial está infectada con esta bacteria, la prevalencia de la infección es alta en poblaciones con alta incidencia de cáncer gástrico, como Japón y Colombia; sin embargo, existen poblaciones con altas prevalencias de infección y muy bajas tasas de cáncer gástrico, hecho que señala que no todas las infecciones con $\mathrm{H}$. pylori incrementan el riesgo de cáncer gástrico $(2,3)$. La infección induce inicialmente una gastritis crónica superficial que, en 15 a 20\% de individuos, puede progresar hacia úlcera péptica o hacia gastritis crónica atrófica, metaplasia intestinal, displasia y carcinoma gástrico o hacia linfoma MALT (4). No se conocen los factores que determinan los resultados tan disímiles que puede tener la infección, ni porqué sólo una minoría de individuos infectados desarrollará enfermedad grave.

Varios genes de $H$. pylori se han asociado con virulencia y mayor riesgo de enfermedad gástrica grave, en particular se han estudiado genes relacionados con la producción de citotoxina bacteriana, la inducción de citocinas proinflamatorias y la adhesión de la bacteria al epitelio (5). El gen vacA codifica la citotoxina vacuolizante VacA; está presente en todas las cepas de H. pylori; las cepas que portan el tipo VacA s1 son más virulentas, tienen mayor actividad citotóxica y se asocian con mayor riesgo de úlcera péptica, gastritis atrófica y cáncer $(6,7)$. El gen asociado a la citotoxina (cagA) es un marcador de la presencia de un islote de patogenicidad (cag PAl) de $40 \mathrm{~kb}$; las cepas cagA positivas se asocian con mayor inducción de interleucina 8 (IL-8) en el

\footnotetext{
Correspondencia:

María Mercedes Bravo, Instituto Nacional de Cancerología. Calle 1a. No. 9-85 Bogotá, Colombia. Teléfono: (571) 334 0959; fax: (571) 3341360

e-mail: mbravo@incancerologia.gov.co

Recibido:28/12/04; aceptado: 12/05/05
}

epitelio y mayor riesgo de úlcera péptica, gastritis atrófica y cáncer $(8,9)$. En estudios posteriores se han encontrado inconsistencias en estas asociaciones, su existencia depende de la población o zona geográfica de la que provengan los aislamientos $(10,11)$. En un estudio reciente con cepas colombianas encontramos que en gastritis no atrófica existe una frecuencia más baja de cepas con genotipos citotóxicos (cagA positivo vacA positivo) en comparación con la observada en gastritis atrófica, metaplasia intestinal, úlcera péptica y cáncer gástrico (12). Esto sugiere que, inclusive en Colombia, un país con alta prevalencia de infección, estos genes podrían ser usados como marcadores de mayor virulencia y riesgo de desarrollar enfermedad gástrica severa; sin embargo, carecen de utilidad para diferenciar cepas con potencial carcinogénico de cepas con potencial ulcerogénico.

Se han propuesto otros genes como marcadores de virulencia. Se ha postulado el gen cagE, que hace parte del cag PAI, como indicador de la funcionalidad de este complejo; este y otros genes del islote son importantes en el incremento de expresión de IL-8 en el epitelio gástrico (13). El gen oipA, localizado fuera del PAI, puede inducir también liberación de IL-8 en la mucosa (14). La presencia del gen $b a b A 2$, que codifica una adhesina BabA2 que se une a antígenos Lewis $b$ de alta expresión en epitelio gástrico, se asocia con mayor riesgo de úlcera péptica, adeno-carcinoma gástrico distal y gastritis con alto grado de actividad (15); se han reportado también variaciones en la prevalencia de cepas babA2 positivas entre países occidentales y asiáticos $(15,16)$.

El propósito de este estudio fue determinar las posibles asociaciones entre la presencia de los genes de virulencia vacA, cagA cagE, babA2 y oipA (o combinaciones de estos) en aislamientos de $H$. pylori de una muestra de pacientes colombianos y las diferentes consecuencias clínicas de la infección. 


\section{Materiales y métodos}

\section{Pacientes y aislamientos bacterianos}

Se evaluaron 166 aislamientos de H. pylori, 128 obtenidos a través del programa de tamizaje en cáncer gástrico del Instituto Nacional de Cancerología, cuyos genotipos cagA y vacA fueron determinados en un estudio previo (12) y 38 aislamientos obtenidos a partir de biopsias gástricas provenientes de pacientes que asistieron a la consulta de endoscopia del Instituto de Diagnóstico Médico, IDIME (Bogotá, Colombia) por síntomas de dispepsia. Todas las cepas se obtuvieron de pacientes que presentaron diagnóstico endoscópico de úlcera péptica (úlcera duodenal o úlcera gástrica) o diagnóstico histopatológico de gastritis crónica atrófica, gastritis crónica sin atrofia, metaplasia intestinal y adenocarcinoma gástrico.

Todos los individuos consintieron su participación por escrito. Se excluyeron los aislamientos de pacientes que hubieran recibido tratamiento de erradicación de $H$. pylori o inhibidores de la bomba de protones durante el mes previo a la toma de muestra y de pacientes con diagnóstico de adenocarcinoma gástrico que hubieran recibido tratamiento de radioterapia o quimioterapia. El protocolo de investigación fue revisado y aprobado por el Comité de Ética del Instituto Nacional de Cancerología.

Mediante endoscopia se tomaron de cuatro a seis biopsias de mucosa gástrica antral. En los pacientes con cáncer, las biopsias se tomaron en zonas no comprometidas por la lesión. La evaluación histológica se hizo sobre cortes histológicos coloreados con hematoxilina-eosina, según los parámetros del sistema de Sydney modificado (17). El diagnóstico de úlcera péptica se basó en la evidencia endoscópica. En los casos en los que se presentó más de una patología se escogió la más grave como diagnóstico de referencia (metaplasia $>$ gastritis atrófica $>$ gastritis crónica).

\section{Aislamiento y cultivo bacteriano}

Dos o tres biopsias se sumergieron en caldo Brucella con glicerol al $20 \%$ y se mantuvieron a $4^{\circ} \mathrm{C}$ hasta 24 horas o a $-20^{\circ} \mathrm{C}$ hasta 72 horas después de la toma de la muestra. Las biopsias se maceraron en condiciones asépticas y fueron cultivadas sobre placas de agar H. pylori (LabM, 140 ), con suplemento de suero de caballo al $8 \%$, isovitalex al $1 \%$ y suplemento selectivo para Campylobacter (Merck). Las placas se incubaron en atmósfera microaerofílica $\left(12 \% \mathrm{CO}_{2}, 5 \% \mathrm{O}_{2}\right)$ a $37^{\circ} \mathrm{C}$ hasta 10 días, según protocolos previamente estandarizados (18). El crecimiento de colonias pequeñas, uniformes, translúcidas, brillantes, ureasa y catalasa positivas, visualizadas con tinción Gram como bacilos largos o curvos Gram negativos indicó la presencia de H. pylori.

\section{Extracción del ADN genómico}

Las bacterias cultivadas en medio sólido se resuspendieron en solución amortiguada fosfato salino (PBS), se ajustaron a una concentración de $5 \times 10^{8}$ bacterias $/ \mathrm{ml} \mathrm{y}$, posteriormente, se centrifugaron a $10.000 \mathrm{rpm}$ durante 10 minutos. El ADN genómico se extrajo mediante tratamiento del precipitado bacteriano con DNAzol (Gibco BRL) según las instrucciones del fabricante.

\section{Tipificación de factores de virulencia}

La determinación de los genotipos de $H$. pylori se basó en la amplificación de los genes vacA, cagA, cagE, oipA y babA2 mediante PCR, de acuerdo con protocolos descritos previamente $(7,14,19-22)$. Los iniciadores para la amplificación de estos genes se presentan en el cuadro 1. Cada reacción de PCR contenía solución tampón PCR (Tris $\mathrm{HCl}$ $20 \mathrm{mM} \mathrm{pH} 8,4, \mathrm{KCl} 50 \mathrm{mM}, \mathrm{MgCl}_{2} 2,5 \mathrm{mM}, 0,2 \mathrm{mM}$ de cada desoxinucleotido (dNTPs) y 0,625 $U$ de TaqDNA polimerasa (Gibco BLR) en un volumen final de $25 \mu \mathrm{l}$ ) y de 1 a $2 \mu \mathrm{l}$ de ADN genómico. Para la amplificación de los genes babA2 y cagE se realizó una denaturación inicial de 4 minutos a $94^{\circ} \mathrm{C}$, seguida de 35 ciclos de: 1 minuto a $94^{\circ} \mathrm{C}, 1$ minuto a $50^{\circ} \mathrm{C}$ y 1 minuto a $72^{\circ} \mathrm{C}$, con un paso final de extensión de 4 minutos a $72^{\circ} \mathrm{C}$. Para la amplificación del gen oipA se realizó una denaturación inicial de 4 minutos a $94^{\circ} \mathrm{C}$, seguida de 40 ciclos de 1 minuto a $95^{\circ} \mathrm{C}, 1$ minuto a $55^{\circ} \mathrm{C}$ y 1 minuto a $72^{\circ} \mathrm{C}$, con un paso de extensión final de 4 minutos a $72^{\circ} \mathrm{C}$. Las condiciones de PCR para los alelos s1/s2, m1 y m2 del gen vacA y para el gen cagA fueron descritas en el estudio 
Cuadro 1. Iniciadores usados para la amplificación de los factores de virulencia de H. pylori.

\begin{tabular}{|c|c|c|c|}
\hline $\begin{array}{l}\text { Gen } \\
\text { (alelo) }\end{array}$ & Iniciador & Secuencia 5'-3' & $\begin{array}{l}\text { Tamaño y ubicación } \\
\text { del producto }\end{array}$ \\
\hline $\operatorname{vacAs} 1 / \mathrm{s} 2$ & $\begin{array}{l}\text { VA1 }-\mathrm{F} \\
\text { VAI -R }\end{array}$ & $\begin{array}{l}\text { ATggAAATACAACAAACACAC } \\
\text { CTgCTTGAATgCgCCAAAC }\end{array}$ & $\begin{array}{l}\text { s1:259bpa }(797-1055) \\
\text { s2: } 286 b^{c}(284-569)\end{array}$ \\
\hline vacA m1 & $\begin{array}{l}\text { VA3 }-F \\
\text { VA3 }-\mathrm{R}\end{array}$ & $\begin{array}{l}\text { GgTCAAAATgCggTCATgg } \\
\text { CCATTggTACCTgTAgAAAC }\end{array}$ & $\begin{array}{l}290 \mathrm{bp} \\
(2741-3030)^{\mathrm{a}}\end{array}$ \\
\hline $\operatorname{vac} A m 2$ & $\begin{array}{l}\text { VA4 }-\mathrm{F} \\
\text { VA4 -R }\end{array}$ & $\begin{array}{l}\text { GgAgCCCCAggAAACATTg } \\
\text { CATAACTAgCgCCTTgCAC }\end{array}$ & $\begin{array}{l}352 \mathrm{bp} \\
(976-1327)^{\mathrm{b}}\end{array}$ \\
\hline $\operatorname{cag} A$ & $\begin{array}{l}\text { CagA-F } \\
\text { CagA-R }\end{array}$ & $\begin{array}{l}\text { TTgACCAACAACCACAAACCGAAg } \\
\text { СТTCССТTAATTgCgAgATTCC }\end{array}$ & $\begin{array}{l}\text { 183bp } \\
(17-199)^{d}\end{array}$ \\
\hline cagE & $\begin{array}{l}\text { CagE-F } \\
\text { CagE-R }\end{array}$ & $\begin{array}{l}\text { TgCTgATACgATTAgAgA } \\
\text { TAgTCCCTTAgTgATgAT }\end{array}$ & $\begin{array}{l}126 \mathrm{pb} \\
(10283-10409)^{\mathrm{e}}\end{array}$ \\
\hline оірA & $\begin{array}{l}\text { oip K25 } \\
\text { oip-R }\end{array}$ & $\begin{array}{l}\text { CAAgCgCTTAACAgATAggC } \\
\text { GCTTCACgAgAAAACgCCTT }\end{array}$ & $(894 \mathrm{pb})^{f}$ \\
\hline babA2 & $\begin{array}{l}\text { babA2F } \\
\text { babA2R }\end{array}$ & $\begin{array}{l}\text { AATCCAAAAAggAgAAAAAgTATgAAA } \\
\text { TgTTAgTgATTTCggTgTAggACA }\end{array}$ & $\begin{array}{l}831 \mathrm{pb} \\
(271-1102)^{\mathrm{g}}\end{array}$ \\
\hline ureC & $\begin{array}{l}\text { ureC-F } \\
\text { urec-R }\end{array}$ & $\begin{array}{l}\text { TgggACTggCgTgAggg } \\
\text { AAgggCgTTTTTAgATTTTT }\end{array}$ & $820 \mathrm{pb}^{\mathrm{h}}$ \\
\hline
\end{tabular}

a. Posiciones nucleotídicas en gen vacA de $H$, pylori $60190(\mathrm{~m} 1)(7)$

b. Posiciones nucleotídicas en gen vacA de H. pylori 87-203(m2) (7)

c. Posiciones nucleotídicas del gen vacA de $H$. pylori Tx30a (7)

d. Posiciones de acuerdo con el ORF cagA en Genbank secuencia L11714 (19)

e. Posiciones nucleotídicas del gen cagE del Genbank AF282853 (20)

f. Posiciones nucleotídicas del gen oipA del Genbank AF233660-AF233683 (14)

g. Posiciones nucleotídicas del gen babA2 del Genbank AF033654 (15)

h. Según metodología de Fujimoto et al. (23)

previo (12). Se amplificó un fragmento de $820 \mathrm{pb}$ del gen ureC para confirmar la calidad del ADN extraído para amplificar fragmentos largos (23). En los aislamientos en los que no se observó una señal clara de amplificación de este gen no se tipificaron los genes babA2 ni oipA puesto que para estos dos genes el producto esperado es de $800 \mathrm{pb}$. Los productos de PCR se visualizaron mediante electroforesis en geles de agarosa al $2 \%$ y tinción con bromuro de etidio, $1 \mathrm{mg} / \mathrm{ml}$, bajo luz ultravioleta.

\section{Análisis estadístico}

Para el análisis estadístico se empleó el programa SPSS versión 9.0, se utilizó la prueba de ji al cuadrado $\left(\chi^{2}\right)$ para comparar las frecuencias de los genes analizados en relación con los diagnósticos histopatológicos. Se empleó la prueba exacta de Fisher cuando fue necesario. Para todos los análisis estadísticos se consideró una diferencia significativa cuando el valor de $p$ era menor de $0,05(p<0,05)$.

\section{Resultados}

Se analizaron 166 aislamientos de $H$. pylori obtenidos a partir de biopsias gástricas de 99 hombres y 67 mujeres, entre 18 y 78 años de edad (media de 49 años); 39 (23,5\%) provenían de pacientes con gastritis no atrófica, $26(15,7 \%)$ de pacientes con gastritis atrófica, $26(15,7 \%)$ de pacientes con metaplasia intestinal $25(15,1 \%)$ de pacientes con cáncer gástrico y $50(30,1 \%)$ de pacientes con úlcera péptica.

Se encontró una mayor prevalencia de cepas con genotipos vacA alelos s1 y m1 (cuadro 2). Cinco por ciento (9/166) de los aislamientos presentó más de un genotipo vacA s1/s2 o m1/m2, en dos aislamientos se detectaron los alelos s1 y s2 y los alelos $\mathrm{m} 1$ y $\mathrm{m} 2$, esto debido probablemente a la existencia de infecciones con múltiples cepas; los aislamientos que presentaron esta característica fueron excluidos de los análisis posteriores. Se corroboró la asociación entre los alelos $\mathrm{s} 1$ y $\mathrm{m} 1$ y entre los alelos $\mathrm{s} 2$ y $\mathrm{m} 2$ del gen vacA pues el 
Cuadro 2. Frecuencia de genotipos vacA, cagA y cagE en 166 aislamientos de $H$. pylori.

\begin{tabular}{llrr}
\hline Gen & \multicolumn{1}{c}{ Alelo } & $\boldsymbol{n}$ & \multicolumn{1}{c}{$(\%)$} \\
\hline vacA & s1 & 116 & $(69,9)$ \\
& s1/s2 & 3 & $(1,8)$ \\
& $s 2$ & 47 & $(28,3)$ \\
& Total & 166 & \\
& $m 1$ & 107 & $(64,5)$ \\
& $m 2$ & 51 & $(30,7)$ \\
& m1/m2 & 8 & $(4,8)$ \\
& Total & 166 & \\
cag PAI & cagA positivo & 121 & $(72,9)$ \\
& cagA negativo & 45 & $(27,1)$ \\
& Total & 166 & \\
& cagE positivo & 124 & $(74,7)$ \\
& cagE negativo & 42 & $(25,3)$ \\
& Total & 166 & \\
oipA & oipA positivo & 102 & $(74,5)$ \\
& oipA negativo & 35 & $(25,5)$ \\
& Total & $137^{*}$ & \\
babA2 & babA2 positivo & 66 & $(48,2)$ \\
& babA2 negativo & 71 & $(52,8)$ \\
& Total & $137^{*}$ & \\
\hline
\end{tabular}

* Se excluyeron 29 cepas en las que no se analizaron oipA y babA2.

$67,5 \%(106 / 157)$ de las cepas presentó genotipo $\mathrm{s} 1 / \mathrm{m} 1$, el $28,7 \%(45 / 157)$ genotipo $\mathrm{s} 2 / \mathrm{m} 2$ y el $3,8 \%(6 / 157)$ vacA s1/m2.

Los genes cagA y cagE se encontraron en el $73 \%$ y $74 \%$ de los aislamientos estudiados (cuadro 2). En el cuadro 3 se presentan las frecuencias de las diferentes combinaciones alélicas para los genes vacA, cagA y cagE. Se observó una asociación importante entre la presencia de los genes cagA y cagE y el genotipo vacA s1m1, pues el 98\% (100/102) de las cepas vacAs1m1 presentó un genotipo cagA positivo/cagE positivo y el 84,6\% (33/39) de los aislamientos vacAs $2 m 2$ presentó un genotipo cag $A$ negativo/cagE negativo $\left(p<10^{-6}\right)$. En este análisis se excluyeron los genotipos heterogéneos vacA s1m2, cagA positivo/cagE negativo y cagA negativo/cagE positivo.

En los aislamientos analizados en este estudio se encontró un predominio de cepas con genotipo vacA s1m1/cagA positivo/cagE positivo (100/157, $63,7 \%$ ). Este genotipo refleja la producción de una citotoxina vacA funcional y la presencia del cagPAI, características asociadas con una mayor virulencia de la bacteria por lo que las cepas con este genotipo se denominaron cepas citotóxicas. $21,0 \%(33 / 157)$ de las cepas analizadas presentó genotipo no citotóxico vacA s2m2/cagA negativo/ cagE negativo; en 15,3\% (24/157) de las cepas estudiadas se encontraron otros genotipos (cuadro 3).

El $75 \%$ de las cepas presentó el gen oipA y el $48 \%$ el gen babA2 (cuadro 2). Se observó una mayor frecuencia del gen oipA en cepas citotóxicas $(81 \%, 69 / 85)$ en comparación con la observada en cepas no citotóxicas (52\%, 13/25) $(p=0,003)$. La prevalencia del gen babA2 en cepas no citotóxicas fue baja $(12 \%, 3 / 25)$, mientras que el 58\% (49/85) de cepas citotóxicas fue babA2 positivo, esta diferencia fue significativa $(p=0,0001)$ (no se muestran los datos).

La distribución de cepas con genotipos citotóxico y no citotóxico en las patologías asociadas con la infección por $H$. pylori se presenta en la figura 1. Se encontró una mayor prevalencia de cepas citotóxicas en pacientes con cáncer (84\%), metaplasia (91\%) y úlcera (81\%) en comparación con la observada en pacientes con gastritis no atrófica $(50 \%)(p=0,002, p=0,008$ y $p=0,007$, respectivamente).

La distribución de babA2 y oipA fue muy similar en todas las patologías (figura 2). Tampoco se observaron diferencias significativas en la

Cuadro 3. Frecuencias de las diferentes combinaciones alélicas encontradas para los genes vacA, $\operatorname{cag} A$ y $\operatorname{cag} E$ en los aislamientos de $H$. pylori.

\begin{tabular}{lccccc}
\hline Gen & $\begin{array}{c}\text { cagA positivol } \\
\text { cagE positivo } \\
\mathbf{n}(\%)\end{array}$ & $\begin{array}{c}\text { cagA negativol } \\
\text { cagE negativo } \\
\mathbf{n}(\%)\end{array}$ & $\begin{array}{c}\text { cagA positivol } \\
\text { cagE negativo } \\
\mathbf{n}(\%)\end{array}$ & $\begin{array}{c}\text { cagA negativol } \\
\text { cagE positivo } \\
\text { n (\%) }\end{array}$ & $\begin{array}{c}\text { Total } \\
\text { (\%) }\end{array}$ \\
\hline s1m1 & $100(94,0)$ & $2(2,0)$ & $1(1)$ & $3(3)$ & 106 \\
s2m2 & $6(13,3)$ & $33(73,3)$ & $2(4,4)$ & $4(9)$ & 0 \\
s1m2 & $5(83,3)$ & $1(16,7)$ & 0 & 0 & 6 \\
\hline
\end{tabular}



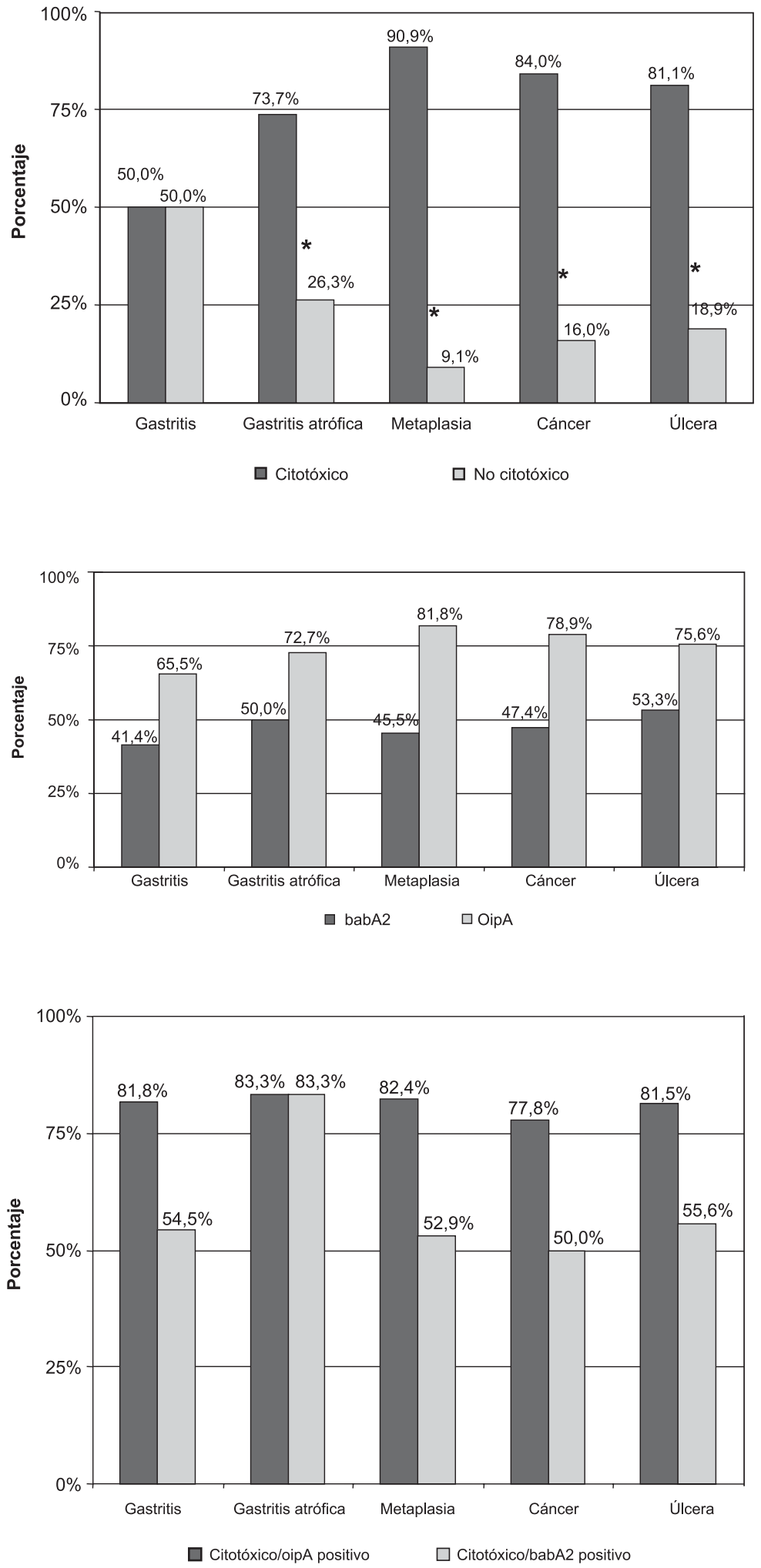

Figura 1. Frecuencia de cepas con genotipo citotóxico (vacAs1m1/cagA positivas/cagE positivas) y no citotóxico (vacAs1m1/cagA negativas/cagE negativas) en las diferentes patologías gastroduodenales.

* $p<0,005$

Figura 2. Frecuencia de los genotipos babA2 y oipA en diferentes patologías gastroduodenales.

Figura 3. Frecuencia de cepas citotóxicas oipA positivas o babA2 positivas en las diferentes enfermedades gastroduodenales. 
frecuencia de cepas citotóxicas oipA positivas o babA2 positivas entre las diferentes enfermedades gastroduodenales (figura 3 ).

\section{Discusión}

En los aislamientos de $H$. pylori caracterizados en este estudio se encontró una alta frecuencia de cepas cagA positivo y cagE positivo (73\% y $74 \%$, respectivamente) y una asociación importante entre estos dos genes, lo que sugiere que la mayoría de los aislados de esta población tiene un islote PAI-cag completo y potencialmente funcional. 3,6\% de las cepas presentó un islote de patogenicidad atípico, frecuencia similar a la reportada en poblaciones de Francia (24), Japón (25), Costa Rica (26), México (27) y Colombia $(12,21,28)$. Debido al bajo número de casos, un eventual papel patogénico de cepas con estos genotipos particulares, no se puede estimar con certeza. Al igual que en estudios anteriores, la presencia del islote de patogenicidad cag se correlacionó con una mayor actividad citotóxica definida por el genotipo vacA s1/m1 (29,30).

Los análisis de secuencia genómica de $H$. pylori han revelado una alta proporción (1\%) de genes que codifican proteínas de membrana externa (31). Debido a esto, ha surgido interés en evaluar el papel de estas proteínas en la patogénesis asociada a la bacteria. Una de estas moléculas es una proteína proinflamatoria de $34 \mathrm{kd}$ codificada por el gen oipA (14). La presencia de este gen en cepas asiáticas se asocia con la presencia de cagA y con inducción de altos niveles de IL-8 en cocultivos con células epiteliales (14), lo que sugiere un posible papel de oipA en la virulencia bacteriana. En este trabajo, la prevalencia del gen oipA fue alta $(74,5 \%)$ y su presencia, sólo o en combinación con cagA y vacA, no se correlacionó con el resultado clínico. Algunos investigadores han descrito dentro del gen oipA una región en la secuencia señal que presenta repeticiones CT (14). La presencia de un número determinado de repeticiones es necesaria para la síntesis de la proteína, por lo tanto, es un indicativo de la funcionalidad del gen. En un reporte reciente, Ando et al. no encontraron diferencias en los patrones de repeticiones $\mathrm{CT}$ en la secuencia señal del gen oipA en aislamientos de $H$. pylori de Holanda y el resultado clínico de la infección (32). Estos autores encontraron que el $96 \%(89 / 93)$ de las cepas cagA positivas presentaba el gen oipA funcional, mientras que ninguna de las 16 cepas cagA negativas tenía el gen oipA funcional. En nuestro estudio encontramos una alta prevalencia de cepas cagA positivo/oipA positivo (81\%), lo que sugiere que la mayoría de cepas citotóxicas tiene el gen oipA funcional; sin embargo, el 52\% (13/26) de las cepas cagA negativas portaba el gen oipA, probable-mente en muchas de estas el gen no sea funcional. Recientemente, Yamaoka (33) evaluó una población de cepas de Estados Unidos y Colombia y encontró que entre varios factores de virulencia sólo el gen oipA funcional se asociaba con mayor densidad de la bacteria, mayor inflamación y presencia de úlcera duodenal. Sin embargo, la presencia de oipA fue relativamente alta en gastritis (64\%) en comparación con las frecuencias observadas en úlcera (88\%) y cáncer (83\%). Considerando nuestros resultados y los estudios de Ando y Yamaoka, la utilidad de oipA como marcador de virulencia es limitada.

La adherencia de $H$. pylori al epitelio gástrico juega un papel clave en la liberación eficiente de factores de virulencia (34). Gerhard et al. (15) reportaron que la presencia de babA2 podría ser un buen indicador de la habilidad de las bacterias para expresar la adhesina de unión a antígenos lewisB (BabA); además, encontraron una asociación significativa entre la detección de babA2 en aislamientos provenientes de la población alemana y la presencia de úlcera duodenal y adenocarcinoma gástrico. En un estudio posterior, Prinz et al. (35) reportaron una importante correlación entre el genotipo vacAs1/cagApositivo y babA2, ya que el $81 \%$ de cepas cagApositivas/vacA s1 portaban también babA2. En nuestro estudio, la frecuencia de babA2 no fue tan alta (48\%), no se correlacionó con la presencia de cagA o vacAs1 pues apenas el $57 \%$ de las cepas citotóxicas portaba babA2; ni se asoció con alguna patología. Estos resultados son similares a los reportados por Kim et al. (36), quienes evaluaron el genotipo babA en cepas coreanas y no encontraron correlación con mayor riesgo de enfermedad. En Japón se reporta una prevalencia de babA2 mucho mayor $(84,9 \%)$, 
sin asociación significativa entre su presencia y el resultado clínico de la infección (37).

En contraste con nuestros resultados, recientemente Zambon et al. (38) analizaron la asociación de babA2 con gastritis, úlcera péptica o metaplasia intestinal en 167 individuos infectados en Italia. Aunque se detectó babA2 sólo en el 36\% de las cepas, su presencia se asoció significativamente con cagA, vacA y oipA. En este estudio las cepas con genotipo babA2 positivo, cagA positivo y vacA $s 1 m 1$ se asociaron con un mayor riesgo de desarrollar metaplasia intestinal, mientras que en individuos portadores de cepas con genotipo cagA negativo, babA2 negativo, vacA s2m2 esta condición se presentó rara vez $(<10 \%)$. Oliveira et al. (39) analizaron los genes babA2 y cagA en cepas de $H$. pylori provenientes de 208 pacientes de Brasil con cáncer gástrico, úlcera duodenal o gastritis. La frecuencia de cepas babA2 $(46,1 \%)$ y cagA $(79,8 \%)$ fue similar a la de nuestro estudio; sin embargo, reportan una asociación fuerte e independiente entre la presencia babA2 y un mayor riesgo de cáncer gástrico y úlcera péptica, pero el valor de riesgo relativo para las cepas cagA positivas fue mayor que el de babA2 (39). En una población de China con alta incidencia de cáncer gástrico, la frecuencia de cepas babA2 positivas fue del $79,8 \%$, y se asoció con inflamación crónica intensa, atrofia glandular y metaplasia intestinal en el antro y con aumento en la proliferación celular epitelial (16). Los resultados de estos estudios sugieren que la expresión diferencial de la adhesina babA2 podría correlacionarse con las diferencias en incidencia de cáncer gástrico en diferentes regiones geográficas. Yamaoka et al. reportan relación entre la presencia de babA2 y con cambos histológicos como metaplasia intestinal y atrofia $(33,40)$; sin embargo, no logran mostrar su utilidad como marcador de riesgo de enfermedad. En conjunto, estos resultados no soportan la hipótesis de que la tipificación de babA2 aumente el poder para discriminar entre cepas cagA positivas aquellas con potencial ulcerogénico o carcinogénico en nuestra población.

En conclusión, en este trabajo se evaluaron varios de los genes que se expresan diferencialmente entre cepas y que, por lo tanto, podrían conferir una mayor capacidad de adaptación a la bacteria $y$, eventualmente, favorecer el desarrollo de cáncer gástrico o úlcera péptica. No se encontraron evidencias que sugieran que los genes babA2 o oipA puedan servir como marcadores de ulcerogénesis o carcinogénesis en esta población, solos o en asociación con cagA, cagE o vacA.

\section{Agradecimientos}

Los autores agradecen al doctor Oscar Gutiérrez del Departamento de Medicina Interna de la Universidad Nacional por su colaboración en la obtención de las muestras.

\section{Conflictos de interés}

Los autores declaran no tener ningún conflicto de interés.

\section{Financiación}

Este trabajo fue financiado por la Embajada del Canadá con fondos de la Carrera Terry Fox, COLCIENCIAS (Contrato 2101-04-11827) y el Instituto Nacional de Cancerología (Recursos de Inversión Código 4103038-15).

\section{Referencias}

1. International Agency for Research on Cancer (IARC). Working group IARC monographs on the evaluation of carcinogenic risk to humans. Schistosomas, liver flukes and Helicobacter pylori. 61. Lyon (France): IARC; 1994.

2. Correa P. Bacterial infections as a cause of cancer. J Natl Cancer Inst 2003;95:E3.

3. Holcombe C. Helicobacter pylori: the African enigma. Gut 1992;33:429-31.

4. Ernst PB, Gold BD. The disease spectrum of Helicobacter pylori: the immunopathogenesis of gastroduodenal ulcer and gastric cancer. Annu Rev Microbiol 2000;54:615-40.

5. Atherton JC. Helicobacter pylori virulence factors. $\mathrm{Br}$ Med Bull 1998;54:105-20.

6. Phadnis SH, Ilver D, Janzon L, Normark S, Westblom TU. Pathological significance and molecular characterization of the vacuolating toxin gene of Helicobacter pylori. Infect Immun 1994;62:1557-65.

7. Atherton JC, Cao P, Peek RM Jr, Tummuru MK, Blaser MJ, Cover TL. Mosaicism in vacuolating cytotoxin alleles of Helicobacter pylori. Association of 
specific vacA types with cytotoxin production and peptic ulceration. J Biol Chem 1995;270:17771-7.

8. Censini S, Lange C, Xiang Z, Crabtree JE, Ghiara P, Borodovsky $M$ et al. cag, a pathogenicity island of Helicobacter pylori, encodes type I-specific and disease-associated virulence factors. Proc Natl Acad Sci USA 1996;93:14648-53.

9. Sharma SA, Tummuru MK, Blaser MJ, Kerr LD. Activation of IL-8 gene expression by Helicobacter pylori is regulated by transcription factor nuclear factor-kappa B in gastric epithelial cells. J Immunol 1998; 160:2401-7.

10. Graham DY, Yamaoka Y. Disease-specific Helicobacter pylori virulence factors: the unfulfilled promise. Helicobacter 2000;5(Suppl.1):S3-9.

11. Höcker $\mathbf{M}$, Hohenberger P. Helicobacter pylori virulence factors-one part of a big picture. Lancet 2003;362:1231-3.

12. Cittelly DM, Huertas MG, Martinez JD, Oliveros R, Posso H, Bravo MM et al. Helicobacter pylori genotypes in non atrophic gastritis are different of the found in peptic ulcer, premalignant lesions and gastric cancer in Colombia. Rev Med Chil 2002;130:143-51.

13. Day AS, Jones NL, Lynett JT, Jennings HA, Fallone CA, Beech $\mathbf{R}$ et al. cagE is a virulence factor associated with Helicobacter pylori-induced duodenal ulceration in children. J Infect Dis 2000;181:1370-5.

14. Yamaoka Y, Kwon DH, Graham DY. A M(r) 34,000 proinflammatory outer membrane protein (oipA) of Helicobacter pylori. Proc Natl Acad Sci USA 2000;97: 7533-8.

15. Gerhard M, Lehn N, Neumayer N, Boren T, Rad R, Schepp W et al. Clinical relevance of the Helicobacter pylori gene for blood-group antigen-binding adhesin. Proc Natl Acad Sci USA 1999;96:12778-83.

16. Yu J, Leung WK, Go MY, Chan MC, To KF, Ng EK et al. Relationship between Helicobacter pylori babA2 status with gastric epithelial cell turnover and premalignant gastric lesions. Gut 2002;51:480-4.

17. Dixon MF, Genta RM, Yardley JH, Correa P. Classification and grading of gastritis. The updated Sydney System. International Workshop on the Histopathology of Gastritis, Houston 1994. Am J Surg Pathol 1996;20:1161-81.

18. Cittelly DM. Henao SC, Martínez JD, Orozco O. Detección de Helicobacter pylori en Colombia: Diferentes metodologías aplicadas a su estudio en una población de alto riesgo de Cáncer Gástrico. Rev Colomb Gastroenterol 2001;3:64-9.

19. van Doorn LJ, Figueiredo C, Rossau R, Jannes G, van Asboeck M, Sousa JC et al. Typing of Helicobacter pylori vacA gene and detection of cagA gene by PCR and reverse hybridization. J Clin Microbiol 1998;36:1271-6.
20. Yamaoka Y, Kodama T, Kita M, Imanishi J, Kashima K, Graham DY. Relation between clinical presentation, Helicobacter pylori density, interleukin 1beta and 8 production, and cagA status. Gut 1999;45:804-11.

21. Yamaoka Y, Kodama T, Gutierrez O, Kim JG, Kashima K, Graham DY. Relationship between Helicobacter pylori ice $A, \operatorname{cag} A$, and vacA status and clinical outcome: studies in four different countries. J Clin Microbiol 1999;37:2274-9.

22. Prinz C, Schoniger M, Rad R, Becker I, Keiditsch E, Wagenpfeil S et al. Key importance of the Helicobacter pylori adherence factor blood group antigen binding adhesin during chronic gastric inflammation. Cancer Res 2001;61:1903-9.

23. Fujimoto S, Marshall B, Blaser MJ. PCR-based restriction fragment length polymorphism typing of Helicobacter pylori. J Clin Microbiol 1994;32:331-4.

24. Audibert C, Burucoa C, Janvier B, Fauchere JL. Implication of the structure of the Helicobacter pylori cag pathogenicity island in induction of interleukin-8 secretion. Infect Immun 2001;69:1625-9.

25. Maeda S, Yoshida H, Ikenoue T, Ogura K, Kanai F, Kato $\mathbf{N}$ et al. Structure of cag pathogenicity island in Japanese Helicobacter pylori isolates. Gut 1999;44:33641.

26. Occhialini A, Marais A, Urdaci M, Sierra R, Muñoz $\mathrm{N}$, Covacci A et al. Composition and gene expression of the cag pathogenicity island in Helicobacter pylori strains isolated from gastric carcinoma and gastritis patients in Costa Rica. Infect Immun 2001;69:1902-8.

27. Morales-Espinosa R, Castillo-Rojas G, GonzalezValencia G, Ponce de León S, Cravioto A, Atherton JC et al. Colonization of Mexican patients by multiple Helicobacter pylori strains with different vacA and cagA genotypes. J Clin Microbiol 1999;37:3001-4.

28. Hsu PI, Hwang IR, Cittelly D, Lai KH, El-Zimaity HM, Gutierrez $\mathrm{O}$ et al. Clinical presentation in relation to diversity within the Helicobacter pylori cag pathogenicity island. Am J Gastroenterol 2002;97: 2231-8.

29. van Doorn LJ, Figueiredo C, Sanna R, Plaisier A, Schneeberger $\mathbf{P}$, de Boer $\mathbf{W}$ et al. Clinical relevance of the cagA, vacA, and iceA status of Helicobacter pylori. Gastroenterology 1998;115:58-66.

30. van Doorn LJ, Figueiredo C, Sanna R, Blaser MJ, Quint WG. Distinct variants of Helicobacter pylori cagA are associated with vacA subtypes. J Clin Microbiol 1999;37:2306-11.

31. Tomb JF, White O, Kerlavage AR, Clayton RA, Sutton GG, Fleischmann RD et al. The complete genome sequence of the gastric pathogen Helicobacter pylori. Nature 1997;388:539-47.

32. Ando T, Peek RM, Pride D, Levine SM, Takata T, Lee YC et al. Polymorphisms of Helicobacter pylori 
HP0638 reflect geographic origin and correlate with cagA status. J Clin Microbiol 2002;40:239-46.

33. Yamaoka Y, Kikichi S, El-Zimaity H, Gutierrez O, Osato MS, Graham DY. Importance of Helicobacter pylori oipA in clinical presentation gastric inflammation, and mucosal interleukin 8 production. Gastroenterology 2002;123:414-24.

34. Ilver D, Arnqvist A, Ogren J, Frick IM, Kersulyte D, Incecik ET et al. Helicobacter pylori adhesin binding fucosylated histo-blood group antigens revealed by retagging. Science 1998;279:373-7.

35. Prinz C, Schöniger M, Rad R, Becker I, Keiditsch E, Wagenpfeil S et al. Key importance of the Helicobacter pylori adherence factor blood group antigen binding adhesin during chronic gastric inflammation. Cancer Res 2001;61:1903-9.

36. Kim SY, Woo CW, Lee YM, Son BR, Kim JW, Chae HB et al. Genotyping CagA, VacA subtype, IceA1, and BabA of Helicobacter pylori isolates from Korean patients, and their association with gastroduodenal diseases. J Korean Med Sci 2001;16:579-84.
37. Mizushima T, Sugiyama T, Komatsu Y, Ishizuka J, Kato M, Asaka M. Clinical relevance of the babA2 genotype of Helicobacter pylori in Japanese clinical isolates. J Clin Microbiol 2001;39:2463-5.

38. Zambon CF, Navaglia F, Basso D, Rugge M, Plebani M. Helicobacter pylori babA2, cagA, and s1 vacA genes work synergistically in causing intestinal metaplasia. J Clin Pathol 2003;56:287-91.

39. Oliveira AG, Santos A, Guerra JB, Rocha GA, Rocha AM, Oliveira CA et al. babA2- and cagA-positive Helicobacter pylori strains are associated with duodenal ulcer and gastric carcinoma in Brazil. J Clin Microbiol 2003;41:3964-6.

40. Yamaoka Y, Souchek J, Odenbreit S, Haas R, Arnqvist A, Borén $\mathrm{T}$ et al. Discrimination between cases of duodenal ulcer and gastritis on the basis of putative virulence factors of Helicobacter pylori. J Clin Microbiol 2002;40:2244-6.MM 